\title{
Evaluation of in vitro fertilization outcomes using the FMR1 CGG repeat level and genotypes as a potential marker
}

\section{Charalampos Theofanakis ${ }^{1}$, Eleni Theochari ${ }^{2}$, Depy Mavrogianni ${ }^{3}$, Christianna Sofokleous ${ }^{4}$, Peter Drakakis ${ }^{3}$, Andreas Pampanos ${ }^{2}$, Sofia Kitsiou-Tzeli ${ }^{4}$, Dimitrios Loutradis ${ }^{5}$}

\author{
'Division of Gynecologic Oncology, 1st Department of Obstetrics \& Gynecology, University of Athens, \\ Alexandra Hospital, Athens, Greece, \\ ${ }^{2}$ Department of Genetics, Alexandra Hospital, Athens, Athens, Greece, \\ ${ }^{3}$ Maternity Obstetrics and Gynaecology Department, 'Alexandra' Hospital, University of Athens \\ Medical School, Athens, Greece \\ ${ }^{4}$ Department of Medical Genetics, University of Athens, Athens, Greece \\ ${ }^{5} 1$ st Department of Obstetrics and Gynecology School of Medicine National and Kapodistrian \\ University of Athens, Athens, Greece
}

\section{Correspondence}

Charalampos Theofanakis MD, M.Sc, Ph.D, Michalakopoulou 88, Ilissia, tel: 00306973042478 , email: charalampostheofanakis@yahoo.com

\section{Abstract}

Introduction: The correlation between FMR1 and ovarian function is a relatively new field of research. It has been stated that premutation carriers present with higher rates of premature ovarian failure, compared to the general population. In the present study, we attempted to correlate the distribution of FMR1 CGG level and genotypes with the outcome of in vitro fertilization protocol between good and poor responders. Materials and Methods: Sixty-two infertile women were enrolled in the study and subdivided into two groups; one group of 36 good responders and one of 26 poor responders, according to the Bologna criteria. Good responders presented with $\geq 8$ oocytes retrieved, basal FSH levels $\leq 10 \mathrm{mIU} / \mathrm{ml}$ and peak estradiol (E2) levels of $\geq 1500 \mathrm{pg} / \mathrm{ml}$. Patients in both groups presented with tubal, male and unexplained infertility. Results: The most prevalent genotype in both good and poor responders was the normal type; $46.2 \%$ and $52.8 \%$, respectively. The distribution of genotypes between good and poor responders did not statistically differ ( $p$-value 0.256). Regarding the FMR1 genetic background, infertile women carrying a normal genotype had statistically significant higher mean oocyte maturation rates $(\mathrm{p}=0.026)$. In the whole study population women not carrying a Low Allele in their Genotype had less mean number of days of stimulation, compared to those carrying a low allele ( $p$-value=0.033). Conclusions: The relative small size of the study population allows for only preliminary results. The random X-inactivation could act as a possible modifier of the impact of FMR1 genotypes in the ovary. 
Key words: FMR1 gene; CGG repeat; poor responders; in vitro fertilization

\section{Introduction}

Variability in the sub-fertile population excludes the possibility of a single approach to controlled ovarian stimulation (COS). During the last decade, attention has been drawn to Fragile X Mental Retardation 1 (FMR1) gene and its implication on reproductive medicine. The term "Fragile X Syndrome (FXS)" appeared back in 1969, when a cytogenetic marker demonstrated fragility at the terminal end of the long arm of the X chromosome (Xq27.3) in a percentage of the metaphases ${ }^{1}$. Several years later, researchers have managed to clone the FMR1 gene, while a special mutation was described. This "dynamic mutation" consisted of a CGG, numerically polymorphic triplet that seemed to increase along the generations ${ }^{2-4}$. FXS is the result of the hypermethylation of the expanded CGG repeats adjacent to exon 1 of the FMR1 gene ${ }^{2}$.

As research progressed, a correlation was found between FMR1 gene and premature ovarian failure (POF), a condition that was described as Fragile $\mathrm{X}$ Premature Ovarian Insufficiency (FXPOI) ${ }^{5}$. This refers to an ovarian dysfunction that ranges from normal menses hormonal levels and reduced fertility to severely elevated levels of Follicle Stimulating Hormone (FSH), abnormal or absent menses and drastically reduced fertility ${ }^{6}$. Moreover, FMR1 gene premutation is related to a neurological disorder, named Fragile X-associated Tremor/Ataxia Syndrome (FXTAS) ${ }^{7}$.

The distribution of the CGG repeats in the general population is categorized as common, intermediate, premutation and full mutation alleles. Common alleles usually contain 6-40 CGG repeats, with a well described distribution pattern [8], they are stable and do not expand upon transmission from parent of offspring. Intermediate alleles contain 41-60 CGG repeats and have variable expansion risks.
Premutation alleles present with 55-199 CGG repeats, are usually unmethylated and can expand to the full mutation (>200 CGG repeats) upon transmission from parent to offspring ${ }^{8,3}$.

The correlation between FMR1 and ovarian function became a field of research when it was noted that $24 \%$ of FMR1 premutation carriers presented with premature ovarian failure, compared to $1 \%$ of all women in the general population ${ }^{5.910}$. It was demonstrated that premutation alleles with 59 to 99 CGG repeats are associated with an increased risk of ovarian dysfunction in female carriers ${ }^{10.11}$. Moreover, research on the link of the FMR1 gene mutations and ovarian physiology, ovarian hormones and infertility, led to definition of a new normal CGGn range of 2634 triplets as long as to different genotypes based on whether both, one or no alleles are within this range, named normal, heterozygous and homozygous, respectively ${ }^{13,14}$.

In the present study, using this proposed revised classification, we attempted to correlate the distribution of the FMR1 gene CGG repeat level and genotypes with the outcome of In Vitro Fertilization (IVF) protocol between good and poor responders.

\section{Materials and Methods}

The study was conducted at the IVF Unit of 1st Department of Obstetrics \& Gynecology, Alexandra hospital, University of Athens, Athens, Greece and informed consent was obtained. Sixty-two infertile women were enrolled in the study and subdivided into two groups; one group of 36 good responders and one of 26 poor responders, according to the Bologna criteria ${ }^{15}$. Good responders presented with $\geq 8$ oocytes retrieved, basal FSH levels $\leq 10 \mathrm{mIU} / \mathrm{ml}$ and peak estradiol (E2) levels of $\geq 1500 \mathrm{pg} / \mathrm{ml}$. The mean age of the good responders was $31,58 \pm 0.72$. Twelve of the poor responders were $<40$ years of 
age and had $>2$ previous failed attempts while 14 were $\geq 40$ years of age and had $\geq 1$ previous failed attempts to conceive with IVF. Patients in both groups presented with tubal, male and unexplained infertility.

Data from age, weight, body mass index (BMI), years of infertility, previous IVF attempts and hormonal profile were collected. Parameters of ovarian stimulation (days of stimulation, total dose of FSH and peak E2 levels) and IVF outcome (number of follicles, oocytes retrieved, maturation and fertilization rate, embryo quality and pregnancy rates) were assessed and analyzed. Poor responders $<40$ years and $\geq 40$ years of age followed a long agonist and a short agonist protocol, respectively.

Workflow consisted of peripheral blood aspiration and data collection, DNA extraction, PCR amplification, capillary electrophoresis, software analysis and statistical analysis.

\section{Results}

The distribution curve of FMR1 allele frequencies based on the CGG repeat number of the studied infertile Greek population was found in accordance with Fu's Distribution curve. We found no shift towards higher ends neither in the total sample, nor the good or the poor responders separately. No intermediate or premutation alleles were found. (CGG)28 was the most frequent allele observed in the total sample, good or poor responders $(43.5 \%$, $42.3 \%, 44.4 \%$, respectively) and this was no different

Table 1. Distribution of FMR1 Genotypes among good and poor responders

\begin{tabular}{lcc}
\hline & Good responders n (\%) & Poor responders n (\%) \\
norm & $19(52,8 \%)$ & $12(46,2 \%)$ \\
het & $14(38,9 \%)$ & $8(30,8 \%)$ \\
hom & $3(8,3 \%)$ & $6(23,1 \%)$ \\
total & $36(100 \%)$ & $26(100 \%)$ \\
Fisher's & P-value $=\mathbf{0 , 2 9 8}$ & \\
exact test & & \\
\hline
\end{tabular}

from what is observed in the Greek general population.

The most prevalent genotype in both good and poor responders was the normal type; $46.2 \%$ and $52.8 \%$, respectively (Table 1 ). The distribution of genotypes between good and poor responders did not statistically differ ( $p$-value 0.256 ). Regarding the FMR1 genetic background, infertile women carrying a normal genotype needed less mean days of stimulation, had statistically significant higher mean oocyte maturation rates $(\mathrm{p}=0.026)$ (Tables $2 \& 3$ ) and presented with favorable pregnancy odds. Infertile women carrying a homozygous genotype needed more mean days of stimulation and had the lowest oocyte maturation rates.

The comparative pregnancy odds in this underrepresented subgroup remains inconclusive, as a sample effect cannot be ruled out. Infertile women carrying a heterozygous genotype had $83 \%$ less pregnancy odds compared to those carrying a norm genotype (95\% C.I: 32\%-91\%, p-value=0,038).In contrast, women carrying a homologous genotype did not have statistically significant different pregnancy odds compared to those carrying a normal genotype ( $\mathrm{p}$-value $=0,559$ ) (Table 4).

In the whole study population women not carrying a Low Allele in their Genotype had less mean number of days of stimulation, compared to those carrying a low allele (p-value $=0.033$ ). In the whole study population the presence of a low allele $(\mathrm{CGG}<26)$ was not associated with differences in pregnancy odds ( $p$-value $=0.198$ ).

\section{Discussion}

To our knowledge, these data show for the first time the impact of FMR1 gene distribution on infertile women with good and poor response to controlled ovarian stimulation. Our study showed that there was no shift towards higher ends in the distribution curve neither in the total sample, nor the good 
Table 2. The correlation of FMR1 alleles to the days of stimulation

\begin{tabular}{lcccc}
\hline Genotype & $\mathbf{N}$ & Days of Stimulation marginal Means & Std. Error & p-value \\
norm & 31 & 9,877 & 0,278 & 0,023 \\
het & 22 & 10,943 & 0,334 & 0,023 \\
hom & 9 & 11,118 & 0,514 & 0,023 \\
adjusted for age (GLM model) & & & & \\
\hline
\end{tabular}

or the poor responders separately (table). The most prevalent was the normal genotype in both groups. Women carrying that genotype needed less mean days of stimulation compared to those carrying the homozygous one. Moreover, women carrying the normal or heterozygous genotype had statistically significant higher mean of oocyte maturation rates compared to those carrying the homozygous genotype $(\mathrm{p}$-value $=0.026)$.

Premutation allelic forms of the FMR1 gene are associated with low ovarian reserve and poor ovarian response. It has been demonstrated that intermediate-sized CGG repeats had no negative effect on ovarian stimulation and clinical outcome using a non-confounding model of oocyte donation. Researches concluded that the number of oocytes retrieved and the clinical outcome were not associated with CGG repeats ${ }^{16}$.

Our study showed that women who carried less than 26 CGG triplet repeats, needed more days of stimulation than those with more than 26 repeats. This parameter could serve as a predictive marker to define the optimal ovarian stimulation protocol for each infertile woman. This comes to succession to previous studies that have shown that low FMR1 alleles represent a potential screening tool for women at genetic risk towards premature ovarian senes- cence ${ }^{17}$. Moreover, it has been stated that variations in the levels of serum anti-Müllerian hormone (AMH) were associated with FMR1 CGG below and above $\mathrm{CGGn}=26-34$, and that effect was varied between different age groups ${ }^{18}$.

Success of assisted reproductive technology (ART) is mainly dependent on ovarian response to stimulation. Several factors have been tested and ovarian reserve is probably the most important one, in predicting success rates after IVF protocols [16]. Common practice suggests that as ovarian reserve decreases, gonadotropin dosage increases, in order to achieve the best outcome. Apart from age, increased gonadotropin use has also been correlated with triplet CGG numbers beyond $35^{19}$.

Moreover, CGG triplet repeats were not associated with differences in pregnancy rates between the study groups. It seems that, based on our findings, FMR1 gene could not serve, at least for now, as a predictive marker for implantation and pregnancy rates.

\section{Conclusions}

Conclusively, FMR1 genotypes seem to have both a quantitative (days of stimulation) and a qualitative (maturation rates) effect on IVF outcomes, as well as on pregnancy odds. The FMR1 could be considered as a candidate gene implicated in IVF success.

Table 3. The correlation of FMR1 alleles to oocyte maturation rates

\begin{tabular}{|c|c|c|c|c|}
\hline Genotype & $\mathbf{N}$ & Maturation Rate marginal Means & Std. Error & p-value \\
\hline norm & 31 & 0,744 & 0,028 & 0,026 \\
\hline het & 18 & 0,796 & 0,038 & 0,026 \\
\hline hom & 7 & 0,598 & 0,060 & 0,026 \\
\hline
\end{tabular}


Table 4. The correlation of FMR1 alleles to oocyte maturation rates

\begin{tabular}{|c|c|c|c|c|c|c|}
\hline \multirow[b]{2}{*}{ Binary Logistic Model } & \multirow[b]{2}{*}{ B } & \multirow[b]{2}{*}{ S.E. } & \multirow[b]{2}{*}{ p-value } & \multirow[b]{2}{*}{ OR } & \multicolumn{2}{|c|}{ 95,0\% C.I. for EXP(B) } \\
\hline & & & & & Lower & Upper \\
\hline age & 0,081 & 0,059 & 0,168 & 0,922 & 0,822 & 1,0347 \\
\hline Geno (het) & $-1,774$ & 0,856 & 0,038 & 0,170 & 0,032 & 0,9081 \\
\hline Geno (hom) & $-0,531$ & 0,909 & 0,559 & 0,588 & 0,099 & 3,4935 \\
\hline Constant & 2,067 & 2,055 & 0,315 & 7,900 & & \\
\hline
\end{tabular}

A rationale of building up a multi-genetic, individualized profile with other genes involved in the IVF process is also an option, where FMR1 could prove informative.

The relative small size of the study population allows for only preliminary results, which require confirmation in a larger study population. The random $\mathrm{X}$-inactivation could act as a possible modifier of the impact of FMR1 genotypes in the ovary.

\section{References}

1. Lubs HA. A marker X chromosome. American journal of human genetics. 1969;21(3):231-44.

2. Oberle I, Rousseau F, Heitz D, Kretz C, Devys D, Hanauer A et al. Instability of a 550-base pair DNA segment and abnormal methylation in fragile $\mathrm{X}$ syndrome. Science. 1991;252(5009):1097-102.

3. Verkerk AJ, Pieretti M, Sutcliffe JS, Fu YH, Kuhl DP, Pizzuti A et al. Identification of a gene (FMR-1) containing a CGG repeat coincident with a breakpoint cluster region exhibiting length variation in fragile X syndrome. Cell. 1991;65(5):905-14.

4. Yu S, Pritchard M, Kremer E, Lynch M, Nancarrow J, Baker E et al. Fragile X genotype characterized by an unstable region of DNA. Science. 1991;252(5009):1179-81.

5. Allingham-Hawkins DJ, Babul-Hirji R, Chitayat D, Holden JJ, Yang KT, Lee C et al. Fragile X premutation is a significant risk factor for premature ovarian failure: the International Collaborative POF in Fragile X study--preliminary data. American journal of medical genetics. 1999;83(4):322-5
6. Welt CK. Primary ovarian insufficiency: a more accurate term for premature ovarian failure. Clinical endocrinology. 2008;68(4):499-509. doi:10. 1111/j.1365-2265.2007.03073.x.

7. Hagerman RJ, Leehey M, Heinrichs W, Tassone F, Wilson R, Hills J et al. Intention tremor, parkinsonism, and generalized brain atrophy in male carriers of fragile X. Neurology. 2001;57(1):127-30.

8. Fu YH, Kuhl DP, Pizzuti A, Pieretti M, Sutcliffe JS, Richards $S$ et al. Variation of the $\mathrm{CGG}$ repeat at the fragile $\mathrm{X}$ site results in genetic instability: resolution of the Sherman paradox. Cell. 1991;67 (6):1047-58.

9. Wittenberger MD, Hagerman RJ, Sherman SL, McConkie-Rosell A, Welt CK, Rebar RW et al. The FMR1 premutation and reproduction. Fertility and sterility. 2007;87(3):456-65. doi:10.1016 /j.fertnstert.2006.09.004.

10. Woad KJ, Watkins WJ, Prendergast D, Shelling AN . The genetic basis of premature ovarian failure. The Australian \& New Zealand journal of obstetrics \& gynaecology. 2006;46(3):242-4. doi:10. 1111/j.1479-828X.2006.00585.x.

11. Bodega B, Bione S, Dalpra L, Toniolo D, Ornaghi $F$, Vegetti $W$ et al. Influence of intermediate and uninterrupted FMR1 CGG expansions in premature ovarian failure manifestation. Human reproduction. 2006;21(4):952-7. doi:10.1093/humrep /dei432.

12. Bretherick KL, Fluker MR, Robinson WP. FMR1 repeat sizes in the gray zone and high end of the normal range are associated with premature 
ovarian failure. Human genetics. 2005;117 (4):376-82. doi:10.1007/s00439-005-1326-8.

13. Gleicher N, Weghofer A, Barad DH. Ovarian reserve determinations suggest new function of FMR1 (fragile X gene) in regulating ovarian ageing. Reproductive biomedicine online. 2010;20(6):76875. doi:10.1016/j.rbmo.2010.02.020.

14. Gleicher N, Weghofer A, Lee IH, Barad DH. FMR1 genotype with autoimmunity-associated polycystic ovary-like phenotype and decreased pregnancy chance. PloS one. 2010;5(12):e15303. doi:10.1371/journal.pone.0015303.

15. Ferraretti AP, Gianaroli L. The Bologna criteria for the definition of poor ovarian responders: is there a need for revision? Human reproduction. 2014; 29(9):1842-5. doi:10.1093/humrep/deu139.

16. Lledo B, Guerrero J, Ortiz JA, Morales R, Ten J, Llacer $\mathrm{J}$ et al. Intermediate and normal sized CGG repeat on the FMR1 gene does not negatively affect donor ovarian response. Human reproduction. 2012;27 (2):609-14. doi:10.1093/humrep/der415.
17. Gleicher N, Yu Y, Himaya E, Barad DH, Weghofer A, $\mathrm{Wu}$ YG et al. Early decline in functional ovarian reserve in young women with low (CGGn < 26) FMR1 gene alleles. Translational research : the journal of laboratory and clinical medicine. 2015;166(5):5027 e1-2. doi:10.1016/j.trsl.2015.06.014.

18. Barad DH, Kushnir VA, Gleicher N. The importance of redundancy of functional ovarian reserve when investigating potential genetic effects on ovarian function. Journal of assisted reproduction and genetics. 2016;33(9):1157-60. doi:10. 1007/s10815-016-0762-9.

19. Gleicher N, Weghofer A, Oktay K, Barad D. Relevance of triple CGG repeats in the FMR1 gene to ovarian reserve. Reproductive biomedicine online. 2009;19(3):385-90.

Received 9-5-2018

Revised 27-7-2018

Accepted 18-8-2018 